\title{
Síndrome de Horner contralateral asociado a bloqueo de ganglio estrellado: reporte de caso y revisión de la literatura
}

\author{
A. Cadavid Puentes ${ }^{1}$, F. Bermúdez Guerrero² y M.V. Zea Medina ${ }^{3}$ \\ ${ }^{1}$ Unidad de Dolor. Hospital San Vicente Fundación y Universidad de Antioquia. Medellín, Colombia. \\ ${ }^{2}$ Servicio de Anestesiología y Reanimación. Universidad de Cartagena. Cartagena, Colombia. ${ }^{3}$ Unidad de \\ Dolor. Hospital San Vicente Fundación. Medellín, Colombia
}

Cadavid Puentes A, Bermúdez Guerrero F, Zea Medina MV. Síndrome de Horner contralateral asociado a bloqueo de ganglio estrellado: reporte de caso y revisión de la literatura. Revisión de la literatura. Rev Soc Esp Dolor 2014; 21(4): 226-229.

\begin{abstract}
We describe a case of contralateral Horner's syndrome following stellate ganglion block. A 56 years old man diagnosed with complex regional pain syndrome type II in right upper arm, who developed after the application of local anesthetic guided by ultrasound in proximity to the right stellate ganglion presented left Horner's syndrome.

The contralateral Horner's syndrome following stellate ganglion block is a rare entity. In our practice occurred one of the few cases reported in the literature, which is of great interest for the medical community.
\end{abstract}

Key words: Horner syndrome. Sympathetic block. Autonomic nerve block. Humans. Adult.

\section{RESUMEN}

Se describe un caso de síndrome de Horner contralateral luego de bloqueo de ganglio estrellado. Se trata de un varón de 56 años con diagnóstico de síndrome doloroso regional complejo tipo II en miembro superior derecho quien posterior a la aplicación de anestésico local guiado por ecografía en proximidad al ganglio estrellado derecho presenta síndrome de Horner izquierdo.

El síndrome de Horner contralateral a un bloqueo de ganglio estrellado es una entidad de ocurrencia supremamente rara, en

Recibido: 02-09-13.

Aceptado: 04-12-13. nuestra práctica se presentó uno de los pocos casos reportados en la literatura, de interés para la comunidad científica.

Palabras clave: Síndrome de Horner. Bloqueo simpático. Bloqueo nervioso autónomo. Humanos. Adulto.

\section{INTRODUCCIÓN}

El síndrome de Horner fue descrito por primera vez en humanos en 1869 por Johann Friedrich Horner (18311886), aunque Claude Bernard (1813-1878) ya había llevado a cabo una descripción fisiológica del simpático cervical en 1852 a través de estudios experimentales en animales (1). Se atribuye el nombre compuesto de síndrome de Claude Bernard-Horner a la disputa sobre la descripción del mismo (2). El médico Horner describió un conjunto de signos y síntomas relacionados con la pérdida del control del sistema nervioso simpático en cabeza y cuello, siendo un hallazgo frecuente después de la anestesia regional del cuello o la extremidad superior (3).

Este síndrome, también conocido como paresia oculosimpática, por bloqueo nervioso autónomo, se caracteriza por la tríada clásica de ptosis palpebral debido a la afectación del músculo de Müller, miosis pupilar y enoftalmos, secundario a la interrupción de la inervación simpática del ojo y sus anexos en diferentes niveles. En su forma completa se aprecia anhidrosis facial ipsilateral y puede ser acompañado por heterocromía del iris en caso de lesiones congénitas (1,4-7).

Tras la inyección del anestésico en proximidad al ganglio estrellado, si la técnica se ha desarrollado correctamente se observa la congestión de la conjuntiva ocular y aparece la tríada descrita por Horner, típicamente ipsilateral al bloqueo. En los próximos minutos puede congestionarse la mucosa nasal por vasodilatación y enrojecerse la cara. 
La aparición de estos signos no implica el bloqueo simpático del brazo, pero sí confirma que la solución anestésica compromete el ganglio estrellado $(8,9)$.

La realización del bloqueo del ganglio estrellado con guía ecográfica ha incrementado su seguridad y probablemente una mayor precisión en su ejecución contrario a las técnicas precedentes, un referente anatómico como la fascia del músculo longus colli, en proximidad al ganglio estrellado, es fácilmente visible con ultrasonido, y actualmente la zona blanco para la aplicación del anestésico (10).

Se reporta un caso inusual de síndrome de Horner contralateral al sitio de bloqueo y las posibles implicaciones clínicas y diagnósticas de este fenómeno.

\section{CASO CLÍNICO}

Paciente masculino de 56 años de edad que es remitido a clínica de dolor por trauma en mano y fractura de radio distal derecho de 6 meses de evolución; fue intervenido quirúrgicamente para osteosíntesis, inmovilizado durante un mes en el postoperatorio. Durante su rehabilitación persistió el dolor a nivel de mano derecha, paresia para el agarre, edema y limitación funcional importante. El dolor se intensifica a los movimientos de la mano y se asocia a calambres. Es valorado por fisiatría quien diagnostica un síndrome doloroso regional complejo tipo II.

Al examen físico se encontró un paciente consciente, orientado, alerta, en buen estado general, hemodinámicamente estable, presentando edema, atrofia moderada de interóseos, actitud de extensión de los dedos sin cambios de la temperatura del miembro superior derecho en comparación con el izquierdo con hipoestesia a nivel radial y disestesia generalizadas en la mano derecha.

El paciente recibió manejo inicial para el dolor con tramadol $75 \mathrm{mg}$ vía oral cada 6 horas con rescates de $50 \mathrm{mg}$ interdosis por dolor moderado a severo, acetaminofén 1 g vía oral cada 8 horas y pregabalina $75 \mathrm{mg}$ vía oral cada 12 horas, sin control adecuado.

Por esto, la clínica de dolor propone la realización de bloqueo simpático cervical derecho guiado por ecografía para descartar dolor mantenido por el simpático.

Depués de la valoración preanéstesica, con el paciente en ayunas, verificación de firmas de consentimientos informados y revisión de equipos satisfactoria, se coloca al paciente en decúbito supino en camilla, monitoria básica, se realiza acceso venoso en miembro superior izquierdo, se administra oxigenoterapia con cánula nasal a 2 1/min, y se aplica sedación con midazolam $2 \mathrm{mg}$ intravenosos alcanzando una sedación de acuerdo a la escala de Ramsay 3/6.

Bajo técnica estéril, previa asepsia y antisepsia del área y colocación de campos estériles para el procedimiento a nivel cervical derecho, se inicia abordaje siguiendo la técnica originalmente descrita por Kapral y cols. (11). Bajo guía ecográfica con transductor lineal de $12 \mathrm{~Hz}$, se visualizan las siguientes estructuras: apófisis transversa de C6, arteria carótida, vena yugular, glándula tiroides, entre otras.

Se avanza aguja espinal 22 Gauge por fuera del plano, transtiroideo, a nivel de C6 y durante el avance de la aguja se contactó la apófisis trasversa de C6, por lo cual se debe retirar y redireccionar su punta en sentido ventral, tratando de direccionar la aguja hacia la fascia del músculo longus colli. Bajo visión ecográfica se realiza hidrodisección la cual inicialmente se observa dentro del músculo longus colli y luego se extiende hasta la fascia del mismo. Se aplicó un volumen de $8 \mathrm{ml}$ de bupivacaína 0,25\% simple bajo visión ecográfica observando la dispersión subfacial y siempre extravascular, en sentido lateral y medial, por debajo de la arteria carótida. Se retira aguja intacta.

El paciente permanece monitorizado en observación y 10 minutos posteriores al procedimiento se observa ptosis e inyección conjuntival, asociado a miosis en ojo izquierdo, contralateral a la zona del bloqueo (Fig. 1).

Adicionalmente a lo anterior se observó aumento de la temperatura del miembro superior ipsilateral al bloqueo y disfonía. Tras 2 horas de la aplicación del anestésico, el paciente mejora de la disfonía, se prueba vía oral satisfactoria y es dado de alta con instrucciones.

\section{DISCUSIÓN}

El síndrome de Horner es el resultado de la parálisis de la cadena simpática cervical ipsilateral (ganglio estrellado o cervicotorácico) causado por cirugías de cuello, anestésicos locales, congénito, compresión local por hematoma o un tumor, o como consecuencia de un inadecuado posicionamiento perioperatorio (12).

Después de una búsqueda sistemática en las bases de datos de Pubmed desde 1960 hasta el año 2012, con las

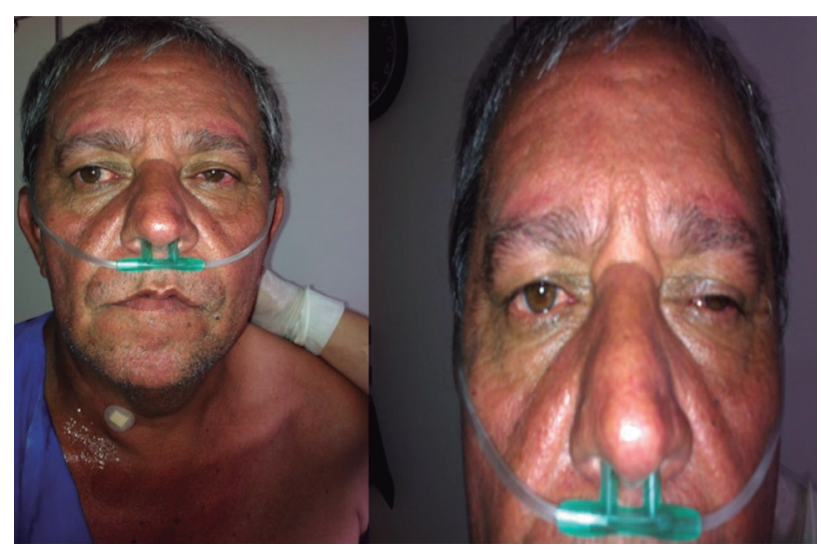

Fig. 1. Sitio de la punción a nivel cervical derecho, se aprecia miosis, ptosis e inyección conjuntival en ojo izquierdo, contralateral al sitio de bloqueo del ganglio estrellado. 
palabras claves "bloqueo simpático, síndrome de Horner, bloqueo simpático cervical", encontramos un primer reporte de caso de bloqueo de ganglio estrellado contralateral en el año de 1969 por Warrick, quien realizó un bloqueo a ciegas durante el tratamiento de un síndrome de Ménière (13). En el año de 1986 se reportó un caso similar, en el que tras un bloqueo del ganglio estrellado izquierdo bajo técnica a ciegas realizado en un paciente adulto de 63 años para el tratamiento de una distrofia simpática refleja, se produjo un síndrome de Horner derecho (14). Otro hallazgo relacionado con la inyección de anestésico local en el ganglio estrellado es la ocurrencia de un síndrome de Horner bilateral, reportado por Joon K. (15).

Warrick trató de dar una explicación al evento sucedido considerando que tal vez el uso de grandes volúmenes de anestésico local o una angulación medial de la aguja podría ser la responsable. En nuestro caso al igual que en el caso reportado por Allen, el volumen administrado de anestésico local fue de $8 \mathrm{ml}$ y la inserción de la aguja se realizó perpendicular al plano frontal.

Sin embargo observamos que durante la hidrodisección hubo inicialmente una mayor dispersión del anestésico en sentido medial en proximidad a la tráquea, en el interior del músculo longus colli, por encima de la apófisis transversa de C6, hecho que ameritó un reposicionamiento. Idealmente la hidrodisección debe lograrse entre la fascia prevertebral y el músculo longus colli, acorde a la técnica recomendada por autores como Narouze (10) (Fig. 2).

No obstante lo anterior, el aumento de temperatura observado en nuestro caso en la extremidad afectada, ipsilateral al sitio de bloqueo, nos lleva a pensar que se logró algún grado de bloqueo del simpático en los primeros ganglios torácicos, correspondientes al miembro superior.

Una explicación posible para nuestro caso de síndrome de Horner contralateral a la zona de punción es una posible mayor difusión medial del anestésico hacia el ganglio estrellado contralateral, por delante del cuerpo vertebral de C6. Esta dispersión inicialmente por fuera del blanco ideal (la fascia del músculo longus colli) y la necesidad de reposicionar la aguja, es un hecho que pudo presentarse por la necesidad de nuevas curvas de aprendizaje para los bloqueos guiados por ecografía, en relación a técnicas precedentes para el bloqueo del ganglio estrellado, como la guía fluoroscópica.

Por otro lado la variabilidad de la anatomía descrita por algunos autores para el ganglio estrellado, definida como plexiforme y que implica un entrecruzamiento, también puede considerarse $(12,16)$. Lo anterior puede ocurrir de forma similar al entrecruzamiento conocido en el plexo celiaco, mesentérico e hipogástrico superior, donde claramente las fibras preganglionares se entrecruzan.

Si bien la presencia de un síndrome de Horner confirma un correcto bloqueo del ganglio estrellado, para el caso del miembro superior se requiere un bloqueo que comprometa fibras de los primeros ganglios simpáticos torácicos y se deben buscar otros signos de interrupción de la actividad simpática tales como aumento de la temperatura mayor a $1,5^{\circ} \mathrm{C}$, para confirmar el bloqueo simpático de la extremidad $(8,9)$.

Con el presente reporte de caso encontramos que un síndrome de Horner contralateral puede asociarse a la realización del bloqueo del ganglio estrellado, sin que se hayan
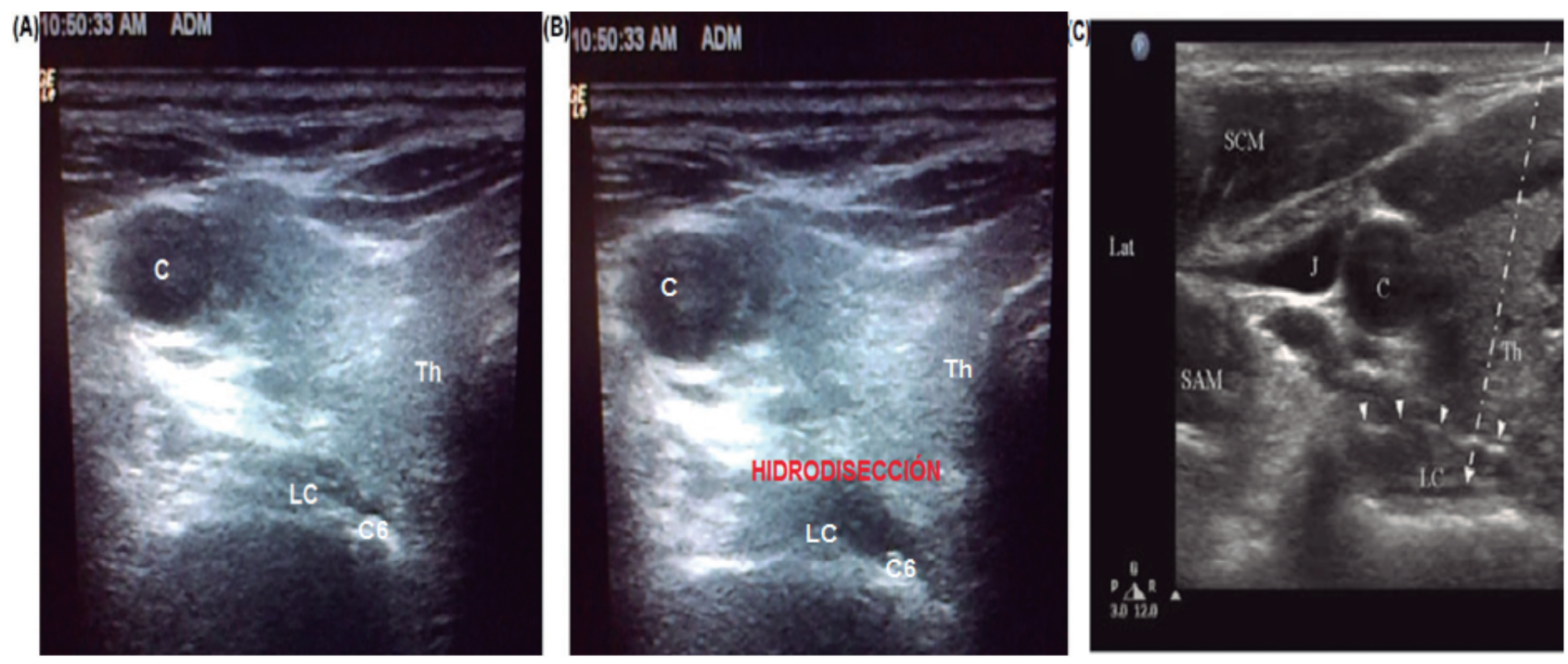

Fig. 2. A. Imagen ecográfica con referencias anatómicas para la identificación del ganglio estrellado derecho. B. Hidrodisección en zona del bloqueo. C. Técnica de Narouze (referencia 10). Plano entre fascia prevertebral y musculo longus colli (flechas sólidas) (C: carótida; J: vena yugular; LC: longus colli; SCM: músculo esternocleidomastoideo; SAM: músculo escaleno anterior; Th: tiroides; C6: sexta vértebra cervical). 
detectado en la literatura preexistente o en nuestro caso eventos adversos mayores asociados.

\section{CORRESPONDENCIA:}

Adriana Cadavid Puentes

Unidad de Dolor

Hospital San Vicente Fundación y Universidad de Antioquia

Medellín, Colombia

e-mail: amcadavidp@gmail.com; franchob2002@hotmail.com

\section{BIBLIOGRAFÍA}

1. González R, Morales C, Obeso S, Longarela Y, García R, Acle L. Horners syndrome after neck surgery. Acta Otorrinolaringol Esp 2012;63:299-302.

2. Ioli P. El Síndrome de Claude Bernard-Horner y otros desconocidos de siempre. Revista del Hospital Privado de Comunidad 2002;5:1-3.

3. Fetzer SJ. Recognizing Homer's Syndrome. Journal of PeriAnesthesia Nursing 2000;15:124-8.

4. Allen AY, Dale RM. Neck procedures resulting in Horner syndrome. Ophthal Plast Reconstr Surg 2009;25:16-8.

5. Asensio VM, Sánchez JC, Macías J, Martínez M. Síndrome de Horner traumático. Arch Soc Esp Oftalmol 2007;82:171-4.

6. Walton KA, Buono LM. Horner syndrome. Curr Opin Ophthalmol. 2003;14:357-63.
7. George A, Haydar A, Adams W. Imaging of Horner's syndrome. Clinical Radiology 2008;63:499-505.

8. López M, De Andrés J. Bloqueo del ganglio estrellado: aplicaciones en el tratamiento del dolor crónico. Rev Soc Esp Dolor 1999;6:449-53.

9. Bonica JJ. Sympathetic nerve blocks for pain diagnosis and therapy. New York: Breon Laboratories; 1984.

10. Narouze SN. Ultrasound-guided interventional procedures in pain management: Evidence-based medicine. Reg Anesth Pain Med 2010;35(Supl. 2):S55-S5.

11. Kapral S, Krafft P, Gosch M, Fleischmann D, Weinstabl C. Ultrasound imaging for stellate ganglion block: Direct visualization of puncture site and local anesthetic spread. A pilot study. Reg Anesth 1995;20:323-8.

12. Salengros JC, Jacquot C, Hesbois A, Vandesteene A, Engelman E, Pandin P. Delayed Horner's syndrome during a continuous infraclavicular brachial plexus block. Journal of Clinical Anesthesia 2007;19:57-9.

13. Warrick JW. Stellate ganglion block in the treatment of Menière's disease and in the symptomatic relief of tinnitus. Br J Anaesth 1969;41:699-702.

14. Allen G, Samson B. Contralateral Horner's syndrome following stellate ganglion block. Canadian Anaesthetists Society Journal 1986;33:112-3.

15. Joon K, Jung Y. Bilateral Horner's Syndrome after a Stellate Ganglion Block. Korean J Anesthesiol 2002;43:241-4.

16. Ataíde A, Brandão B, Pacheco C, Calheiros D, Oliveira $\mathrm{G}$, Neto B, et al. Estudio topográfico y biométrico del ganglio cervicotorácico (ganglio estrellado). Int J Morphol 2008;26:451-5. 